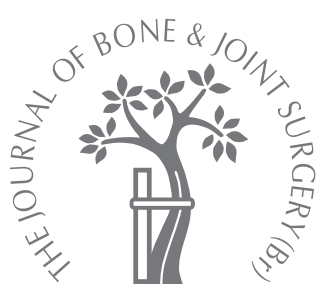

S.-R. Lyu

From Tzu-Chi Dalin General Hospital, Chiayi, Taiwan

\section{Arthroscopic medial release for medial compartment osteoarthritis of the knee}

\author{
THE RESULT OF A SINGLE SURGEON SERIES WITH A MINIMUM \\ FOLLOW-UP OF FOUR YEARS
}

S.-R. Lyu, MD, PhD,

Orthopaedic Surgeon, Chief of Joint Center

Tzu-Chi Dalin General Hospital, Tzu-Chi University, No. 2 MinShen Road, Dalin, Chiayi,

Taiwan.

Correspondence should be sent to $\operatorname{Dr}$ S.-R. Lyu; e-mail: srlyu@seed.net.tw

(C)2008 British Editorial Society of Bone and Joint Surgery doi:10.1302/0301-620X.90B9. $20584 \$ 2.00$

$J$ Bone Joint Surg $[\mathrm{Br}]$ 2008;90-B:1186-92. Received 10 December 2007; Accepted after revision 18 April 2008

\begin{abstract}
The outcome of arthroscopic medial release of $\mathbf{2 5 5}$ knees in 173 patients for varying grades of osteoarthritis involving the medial compartment is reported. All operations were performed by a single surgeon between January 2001 and May 2003. The Knee Society score for pain and the patient's subjective satisfaction were used for the outcome evaluation. Overall, satisfactory outcome was reported for 197 knees $(\mathbf{7 7 . 3} \%)$ and the mean Knee Society score for pain improved from 17.6 (95\% confidence interval, 16.7 to 18.5), preoperatively to 39.4 (95\% confidence interval, 37.9 to 41.1$)(p<0.001)$. There were minor manageable complications of persistent effusion in $\mathbf{1 6}$ knees and prolonged wound discomfort in 11. In total, 15 of the 21 knees with poor results were converted to total knee replacements and two other patients (three knees) were offered this option after a mean period of 16 months.

Based on these observations arthroscopic medial release is an effective treatment for osteoarthritis of the medial compartment of the knee joint and can be expected to reduce the pain in the majority of patients for at least four years post-operatively.
\end{abstract}

Arthroscopic techniques for the management of degenerative conditions of the knee include joint lavage, debridement, ${ }^{1-4}$ abrasion arthroplasty $^{5}$ and microfracture. ${ }^{6,7}$ Nevertheless, their effectiveness has not been proven in prospective trials and the mechanism by which they improve the course of degenerative conditions of the knee has not been established. ${ }^{2,8}$ Therapeutic arthroscopy performed in the osteoarthritic knee also remains a source of controversy because of a lack of a clear consensus on the indications and surgical technique, as well as the unpredictability of the outcome. ${ }^{9-11}$ In 2007 , Siparsky et $\mathrm{al}^{12}$ performed a retrospective, evidence-based review of the literature on the arthroscopic treatment of osteoarthritis of the knee and found limited support for its use in this condition.

Medial release as part of the surgical treatment for medial compartment osteoarthritis of the knee has been reported occasionally since $1940 .{ }^{13-19}$ In 1960 , Loeffler ${ }^{20}$ described a procedure which included resection of the medial collateral ligament for patients with both early and late degenerative changes of the medial compartment. Success rates ranging from $72.5 \%$ to $90 \%$ have been reported following his procedure. ${ }^{21-23}$ In 2001, Leon, Blanco and Guthrie $^{24}$ reported an arthroscopic intervention they referred to as arthroscopic decompressive medial release. They divided the medial third of the capsule and the medial collateral ligament (MCL) transversely $1.5 \mathrm{~cm}$ proximal to the medial meniscus to unload the medial compartment and achieved satisfactory results in a small group of 38 patients. In 2004, Moriya et $\mathrm{al}^{25}$ reported the clinical outcomes of a similar arthroscopic concept of posteromedial release. The medial compartment was released by separating the medial capsule and MCL from the medial tibial plateau after meniscectomy. In their opinion, relatively advanced osteoarthritis of the knee for which arthroscopic debridement has conventionally been contraindicated, could be treated with this procedure in carefully selected patients. However, none of these salvage procedures have become popular.

During arthroscopic debridement in patients with medial compartment osteoarthritis the author has often observed unusual degenerative changes affecting the medial femoral condyle with adjacent inflammatory synovial tissue and a medial plica. The common presence of a plica in these circumstances and its positive correlation in severity to the pathological changes seen on the medial femoral condyle $^{26}$ encouraged its resection with an associated medial release. We hypothesised that the eradication of the interplay between 
the inflamed medial plica and the medial femoral condyle would be beneficial. Subsequently, the procedure has been refined. During the late 1990s it became referred to as arthroscopic medial release and was standardised. In this retrospective study, the details of this procedure and its clinical results are reported.

\section{Patients and Methods}

Between January 2001 and May 2003, 215 patients underwent arthroscopic surgery for osteoarthritis involving the knee at the Tzu-Chi Dalin General Hospital. Within this group of patients, 267 arthroscopic medial releases were performed in 182 patients (153 female and 29 male) with medial compartment osteoarthritis. The mean age of the patients was 61 years (38 to 75 ). A total of 85 patients received bilateral treatment: 79 were treated with simultaneous operations and six were treated after an interval of six to nine months. The procedure was offered to patients with osteoarthritis of any stage that was confined mainly to the medial compartment. All patients had been treated conservatively for more than one year without success prior to the arthroscopic treatment. The exclusion criteria were the presence of modified Outerbridge ${ }^{27}$ grade III or IV osteoarthritis of the lateral or patellofemoral compartments, unstable meniscal fragments, instability due to previous ligament injury, or post-traumatic osteoarthritis. Age, radiological grading according to Kellgren and Lawrence, ${ }^{28}$ and the pain domain of the Knee Society scoring system ${ }^{29}$ (KSS) were recorded pre-operatively.

The anteroposterior standing radiographs of these patients were evaluated for the severity of medial compartment osteoarthritis. The four-grade classification of Kellgren and Lawrence ${ }^{28}$ was used, and is as follows: grade 1, doubtful narrowing of joint space and possible osteophytic lipping; grade 2, definite osteophytes and possible narrowing of joint space; grade 3, moderate multiple osteophytes, definite narrowing of joint space, some sclerosis and possible deformity of bone ends; grade 4, large osteophytes, marked narrowing of joint space, severe sclerosis, and definite deformity of bone ends. The radiological grading was performed by the author, who also performed all of the procedures.

Surgical procedure. All procedures were performed under spinal or general anaesthesia with the use of a tourniquet.

Routine arthroscopic examination was performed through an inferolateral portal and the condition of articular cartilage, menisci and ligaments was evaluated. If osteoarthritis was found to involve the lateral or patellofemoral compartments, unstable meniscal fragments, or ligamentous deficiency was identified, the patient was excluded from the study.

The condition of the medial plica was studied in detail. The type and grade of severity of the plica according to its gross appearance, was classified as previously described. ${ }^{26}$ In summary, a type A plica has no direct contact with the medial femoral condyle, a type B rides onto but not beyond

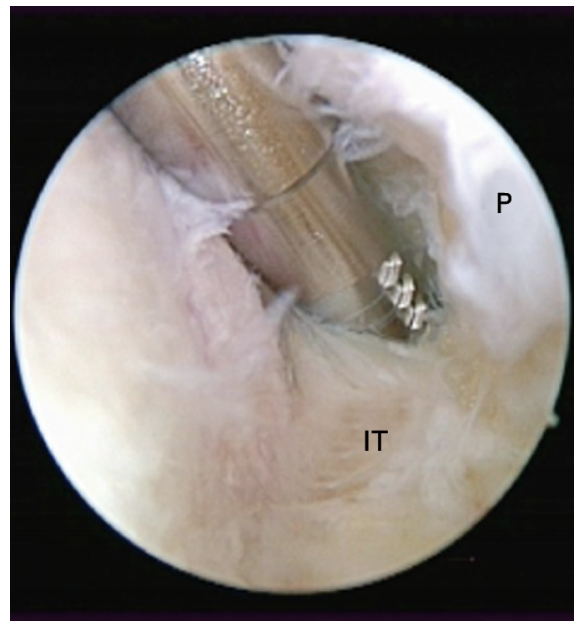

Fig. 1

Intra-operative photograph showing that the debridement is carried out through the superolateral portal as medially as possible to eradicate all the inflammatory tissue (IT) occupying the inferomedial region of the patella $(P)$.

half the width of the medial femoral condyle, and a type C plica extends over more than half of the width of the medial femoral condyle. A grade I plica looks like a membrane, its margin is semi-transparent and is soft in consistency when palpated by a probe. The grade II plica is not transparent but is hypertrophied and thickened while retaining a soft consistency. A grade III plica looks like a fibrotic band and is thicker than a grade II but is elastic in consistency. A grade IV plica exhibits the additional sign of wear (caused by abrasion with the femoral condyle) as well as looking fibrotic. Its margin is frail and fibrillated. A grade $\mathrm{V}$ plica represents an inflamed version of a grade IV, with focal synovitis adjacent to it. In most cases synovectomy is required in this grade to enable clear visualisation of the plica. The observation for the classification of the plica is performed through the inferolateral portal with the knee in full extension.

First-stage debridement. The inflammatory tissue occupying the inferomedial region of the patella, including the ligamentum mucosum, fibrotic or inflamed synovium, capsule and distal part of the medial plica, was removed using a power shaver passed through a superolateral portal, with the debridement extended as medially as possible to eradicate all the inflammatory tissue found in this space (Fig. 1). Release of the medial gutter. The obliterated space of the medial gutter was released by resection of the fibrotic synovium, capsule and proximal part of the medial plica little by little using either an electrical shaver or a punch. When complete, the tightened medial patellofemoral joint had been released and the medial gutter cleared of any fibrotic or inflammatory tissue. In most cases, this stage of the procedure could be performed through the superolateral portal 


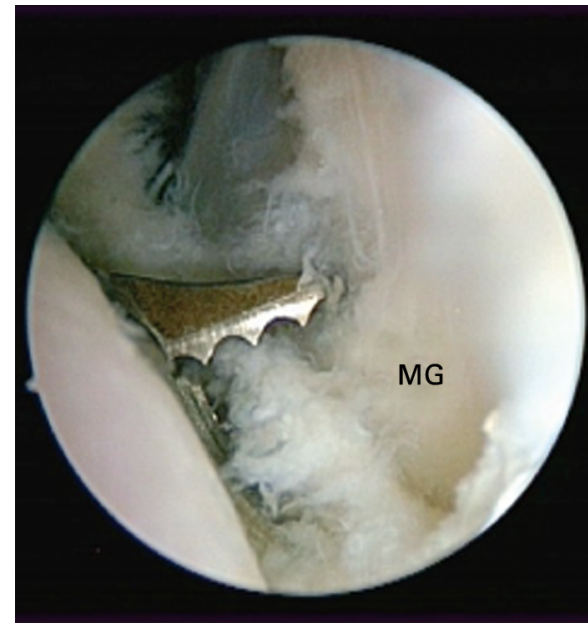

Fig. 2

Intra-operative photograph showing that the obliterated space of the medial gutter (MG) is gradually released by resection of the fibrotic synovium, capsule, and proximal part of the medial plica. In most cases this stage could be performed through the superolateral portal.

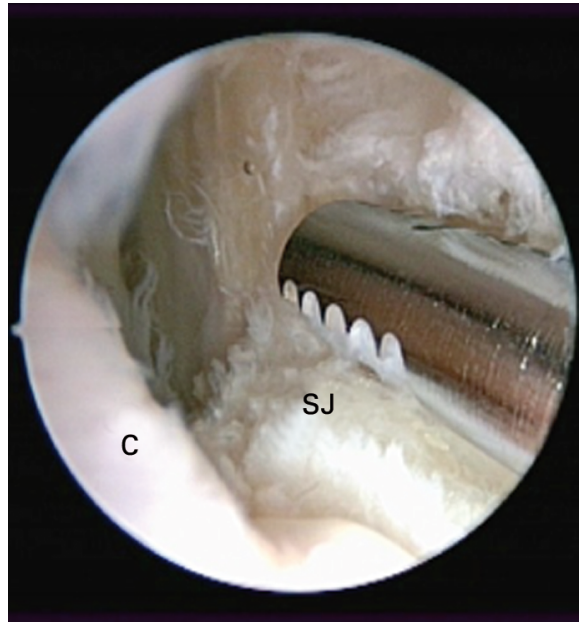

Fig. 3

Intra-operative photograph showing that the edge of the resection over the synovio-meniscal junction (SJ) should be smoothed to avoid impingement against the medial femoral condyle (C) when the knee joint is moves.

(Fig. 2). However, in some difficult cases it was more practical to use an inferomedial portal.

Final assessment of the release. The edge of the resection at the synoviomeniscal junction was smoothed to avoid impingement against the medial femoral condyle when the knee joint was manipulated through a range of movements (Fig. 3). The adequacy of the medial release was confirmed

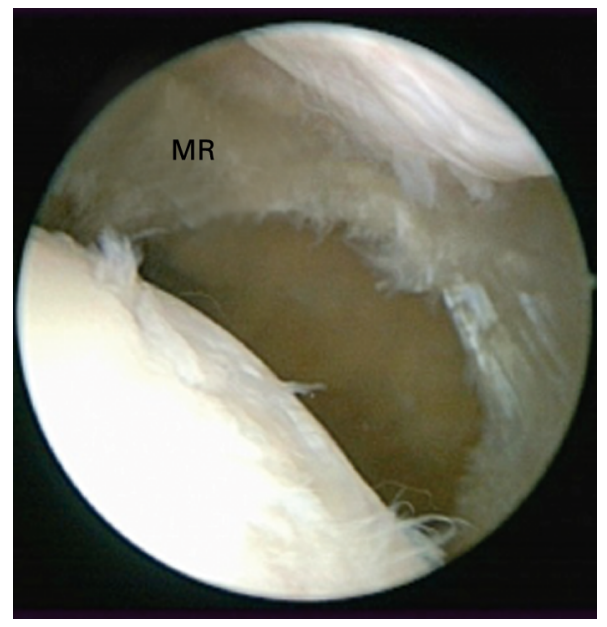

Fig. 4

Intra-operative photograph showing that the adequacy of the medial release is confirmed by passing the arthroscope through the patellofemoral joint and is verified if the medial facet can be easily opened and the medial retinaculum (MR) visualised in full extension.

by passing the arthroscope under the patella and verifying that the previously tightly closed medial patellofemoral joint space could be easily opened and the medial retinaculum visualised when the knee was held in full extension (Fig. 4).

The target of the capsular release was layer III, the socalled 'true capsule' of the three-layer medial structure described by Warren and Marshall. ${ }^{30}$ As shown in Figure 5, the capsulectomy was extended superiorly to the midline of the suprapatellar pouch and inferiorly to the upper margin of medial meniscus. Anteriorly, it was extended to the medial margin of the patella, and posteriorly it was continued until a portion of the conjoined part of layer II and III was removed and the gracillis tendon could be seen. Only the deep medial ligament was severed by this procedure, so that medial stability was not disturbed.

Synovectomy and chondroplasty. Any focal synovitis or loose chondral flaps on the cartilaginous surface were debrided as conventional arthroscopic treatment for osteoarthritis of the knee, but no bony procedure, such as drilling or microfracture, was performed. At the end of the procedure, thorough irrigation was performed to remove any debris from the knee.

Post-operative management. A suction drain was used for 24 hours. The leg was protected by an elastic bandage for one week. The patient was allowed to mobilise freely as comfort permitted without restriction on weight-bearing. The patient was discharged the day after the operation. Active range of movement and quadriceps exercises were encouraged at home.

Follow-up and evaluation of outcome. The patients were reviewed monthly for three months. Thereafter, they 


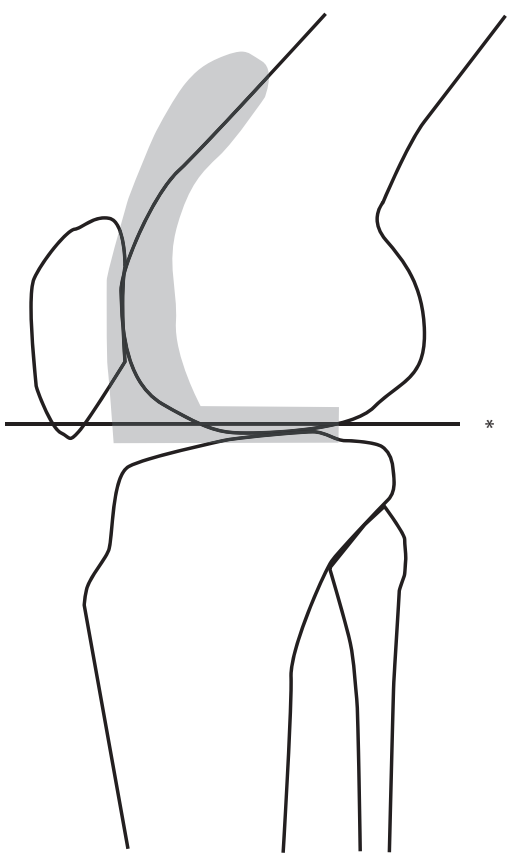

Fig. $5 a$

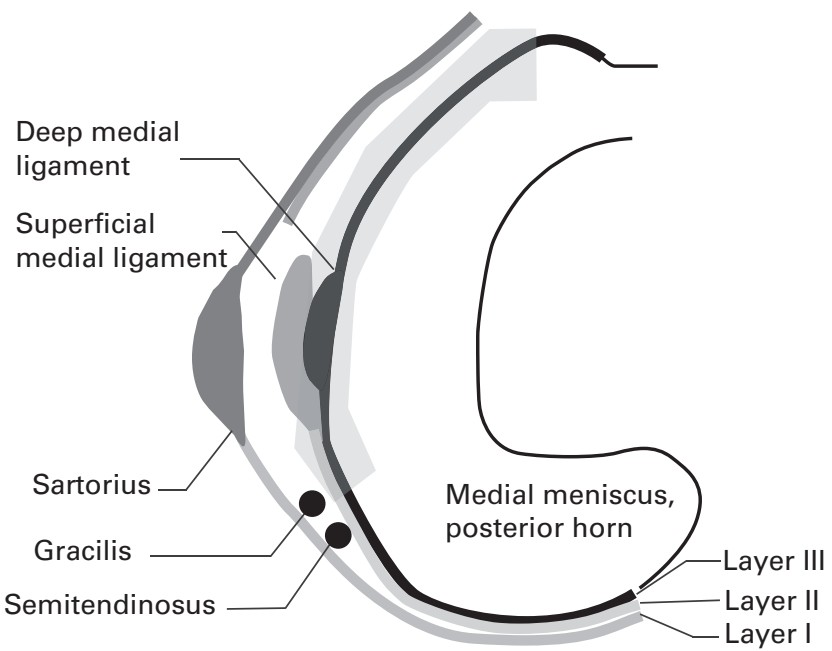

Fig. $5 b$

Diagrams showing a) the extent of the medial capsulectomy in the shaded area of the lateral view of the knee joint. The marked line is the level of cross-section above the meniscus as shown in part b, and b) the shaded area indicates the extent of the capsulectomy above the medial meniscus. Note that only the deep medial ligament is divided. The tendon of gracilis could be seen after the procedure.

returned annually for assessment. The 50-point pain domain of the KSS was used to evaluate the pain both preand post-operatively, where 50 points indicate no pain and 0 points indicate severe pain. ${ }^{29}$ Subjective satisfaction was assessed by direct questioning using a categorical scale prepared for this study (Table I). The outcome was regarded as satisfactory if subjective satisfaction was rated as 'excellent' or 'good'. The inquiry into subjective satisfaction and the evaluation of KSS were conducted by nursing specialists. All investigations focused on individual knees in bilateral cases.

Statistical evaluation. Results were presented as means, SD and $95 \%$ confidence intervals (CI) as appropriate. Comparisons were made using one-way analysis of variance (ANOVA) to detect differences in the distribution of patient age and the type and grade of severity of the medial plica in each grade of osteoarthritis. Comparison of pre- and postoperative Knee Society pain scores was performed using the paired $t$-test. Post-operative Knee Society scores $>40$ and an increase in the score of $>20$ were defined as a successful outcome. Logistic regression tests were used to evaluate the correlation of size of the plica, the pathological severity of the plica and the severity of osteoarthritis with the patient's age. A p-value $<0.05$ was considered to be statistically significant. All statistical analysis was carried out using the statistical discovery software, JMP (version 5.0.1.2, SAS Institute Inc., Cary, North Carolina).

\section{Results}

All the knees in the study were found to have a medial plica. The type of plica and severity of the osteoarthritis were examined in relation to the mean age of the patient. The size of the plica was negatively correlated with the patient's age $(r=0.29, p<0.0001)$. The mean age and distribution of different grades of plica and the type and severity for each grade of osteoarthritis are shown in Table II. The severity of the plica $(\mathrm{r}=0.23, \mathrm{p}=0.0018)$ and the severity of osteoarthritis $(\mathrm{r}=0.58, \mathrm{p}<0.0001)$ were positively correlated with the patient's age.

The mean follow-up period was 5.3 years (4 to 6.5). None of the nine grade I osteoarthritic knees were lost to follow-up; two of 90 knees $(2.2 \%)$ with grade II osteoarthritis in two patients, six of 135 knees (4.4\%) with grade III osteoarthritis in four patients, and four of 33 knees $(12.1 \%)$ with grade IV osteoarthritis in three patients were lost to follow-up. There were 255 knees in 173 patients available with more than four years follow-up.

Post-operative complications. Of the 255 available knees, $16(6.3 \%)$ in 12 patients experienced persistent effusion and discomfort for more than two weeks after the procedure. After initial treatment, including rest, compression bandaging and anti-inflammatory medication, the effusion subsided spontaneously within two weeks in 11 knees. The other five knees in four patients required aspiration of the knee after four weeks. 
Table I. Subjective clinical outcome evaluation criteria

\begin{tabular}{ll}
\hline Outcome & Criteria \\
\hline Excellent & Free of symptoms, no limitation in activities \\
Good & Greatly improved, occasional pain, normal activities \\
Fair & Same as pre-operative condition, no improvement \\
Poor & Has received or considered further operative treatment \\
\hline
\end{tabular}

Table II. Distribution of mean ages and number of knees for each type and grade of plica in relation to each grade of osteoarthritis

\begin{tabular}{|c|c|c|c|c|c|c|c|c|c|c|c|}
\hline & \multicolumn{11}{|c|}{ Severity grading of osteoarthritis } \\
\hline & \multicolumn{2}{|l|}{$\mathrm{I}$} & \multicolumn{2}{|l|}{ II } & \multicolumn{2}{|l|}{ III } & \multicolumn{2}{|l|}{ IV } & \multicolumn{2}{|l|}{ All grades } & \multirow[t]{2}{*}{ p-value } \\
\hline & Age & $\begin{array}{l}\text { Number } \\
\text { of knees }\end{array}$ & Age & $\begin{array}{l}\text { Number } \\
\text { of knees }\end{array}$ & Age & $\begin{array}{l}\text { Number } \\
\text { of knees }\end{array}$ & Age & $\begin{array}{l}\text { Number } \\
\text { of knees }\end{array}$ & Age & $\begin{array}{l}\text { Number of } \\
\text { knees }\end{array}$ & \\
\hline \multicolumn{12}{|l|}{ Plica type } \\
\hline A & & 0 & 59 (49 to 70$)$ & 7 & 66 (53 to 75 ) & 36 & 70 (62 to 75$)$ & 14 & $66^{*}$ (49 to 75$)$ & 57 & \\
\hline B & 43 (38 to 51$)$ & 8 & 55 (38 to 65 ) & 78 & 61 (52 to 70$)$ & 93 & 65 (53 to 74$)$ & 19 & $58^{*}$ (38 to 74$)$ & ) 198 & \\
\hline c & 41 & 1 & 52 (39 to 61$)$ & 5 & 58 (45 to 66$)$ & 6 & 0 & 0 & $54^{*}(39$ to 66$)$ & 12 & $<0.001$ \\
\hline \multicolumn{12}{|c|}{ Plica grade } \\
\hline I & & 0 & & 0 & & 0 & & 0 & & 0 & \\
\hline II & 38 (38 to 39$)$ & 2 & 41 (38 to 46$)$ & 11 & & 0 & & 0 & $40^{*}$ (38 to 46$)$ & 13 & \\
\hline III & 41 (38 to 44$)$ & 2 & 44 (39 to 51$)$ & 14 & 53 (45 to 62$)$ & 22 & & 0 & $49^{*}$ (38 to 62$)$ & 38 & \\
\hline IV & 45 (39 to 51$)$ & 4 & 57 (48 to 63 ) & 8 & 60 (51 to 68 ) & 23 & 63 (56 to 69$)$ & 4 & $58^{*}$ (39 to 69$)$ & 39 & \\
\hline v & 47 & 1 & $60(44$ to 70$)$ & 57 & 64 (45 to 75$)$ & 90 & $67(53$ to 75$)$ & 29 & $63^{*}(44$ to 75$)$ & 177 & \\
\hline All plicae & 43 & 9 & 55 (38 to 70$)$ & 90 & 62 (45 to 75$)$ & 135 & 67 (53 to 75 ) & 33 & 61 (38 to 75 ) & 267 & 0.0002 \\
\hline
\end{tabular}

* statistically significant by one way analysis of variance $(p<0.001)$

Table III. Distribution of subjective outcome for knees (\%) and mean (95\% confidence interval) Knee Society score for pain pre- and post-operatively relative to the osteoarthritis grading

\begin{tabular}{|c|c|c|c|c|c|c|c|c|c|}
\hline \multirow[b]{2}{*}{ Grade } & \multicolumn{5}{|c|}{ Subjective outcome } & \multicolumn{4}{|l|}{ Knee Society score } \\
\hline & Excellent & Good & Fair & Poor & Satisfied (\%) & Pre-operative & Post-operative & Improved & $p$-value (t-test) \\
\hline I & 3 (33.3) & $6(66.7)$ & 0 & 0 & 100 & $21.1(18.5$ to 23.7$)$ & 46.1 (43.5 to 48.7$)$ & $25 \quad$ (21.7 to 28.3$)$ & $<0.001$ \\
\hline II & $58(65.9)$ & $20(22.7)$ & $10(11.4)$ & 0 & 88.6 & 18.7 (17.5 to 20.0$)$ & 46.4 (45.0 to 47.7$)$ & 27.6 (25.4 to 29.8 ) & $<0.001$ \\
\hline III & $29(22.5)$ & $68(52.7)$ & $19(14.7)$ & $13(10.1)$ & 75.2 & 17.5 (16.2 to 18.8 ) & 37.4 (35.1 to 39.8 ) & 20.0 (17.2 to 22.7$)$ & $<0.001$ \\
\hline IV & 0 & $13(44.8)$ & $8(27.6)$ & $8(27.6)$ & 44.8 & 13.5 (10.0 to 16.9$)$ & 25.2 (19.5 to 30.8$)$ & 11.7 (5.5 to 17.9$)$ & $<0.001$ \\
\hline All grades & $90(35.3)$ & $107(42.0)$ & $37(14.5)$ & $21(8.2)$ & 77.3 & 17.6 (16.7 to 18.5$)$ & 39.4 (37.9 to 41.1$)$ & 21.8 (20.0 to 23.7 ) & $<0.001$ \\
\hline
\end{tabular}

Most patients reported some pain, catching or instability during the first post-operative month. This resolved spontaneously. In 11 knees $(4.3 \%)$ in nine patients there was discomfort due to wound irritation for more than three months. In five knees in four patients this settled with routine treatment within three months. A hydrocortisone injection was required in three other knees and two further patients (three knees) had persistent pain and instability despite this treatment. Their symptoms settled, however, following arthroscopy and adhesiolysis. A fibrotic band and tightness of the medial capsule was found in these knees.

Outcome. Of the 255 knees available for final review the subjective assessment was satisfactory in $197(77.3 \%)$. The mean KSS for pain improved from 17.6 (95\% CI 16.7 to 18.5 ) pre-operatively to 39.4 (95\% CI 37.9 to 41.1 ) at final follow-up. The improvement was statistically significant in all groups (Table III). For knees with an excellent outcome, the mean post-operative KSS for pain was 50, with a mean increase of 33.1 (95\% CI 31.8 to 34.4), for knees with a good outcome it was 41.8 (95\% CI 41.2 to 42.4), with a mean increase of 24.9 (95\% CI 23.4 to 26.4), and for knees with a fair outcome it was $26.2(95 \%$ CI 24.6 to 27.8$)$, with a mean increase of 1.9 (95\% CI 0.9 to 2.9$)$. However, for knees with a poor outcome, the mean KSS for pain was 5.2 (95\% CI 3.1 to 7.3 ) pre-operatively with a mean reduction of 6.9 (95\% CI 4.9 to 8.9 ) post-operatively. Applying a post-operative KSS for pain of $>40$ or the increase of scores of $>20$ as the criteria for treatment success, for knees with grade I osteoarthritis the success rate was $100 \%$ (nine knees), for grade II, $87.5 \%$ (77 of 88 knees); for grade III, $62.8 \%$ (81 of 129 knees), and for grade IV, $41.2 \%$ (12 of 
29 knees). Applying this evaluation with the KSS for pain the success rate of the whole series was $70.2 \%$ (179 of 255 knees).

Of the 21 knees with poor results three occurred in two patients who received a second arthroscopy and adhesiolysis. In total, 15 of the 21 knees with poor results were converted to total knee replacement after a mean period of 16 months (13 to 25 ). The other two patients (three knees) were also offered knee replacement. All the patients with poor results had grade III and grade IV osteoarthritic changes.

\section{Discussion}

Arthroscopic release for medial compartment osteoarthritis of the knee gave a satisfactory subjective outcome rate of $77.3 \%$, and the success rate evaluated by KSS for pain was $70.2 \%$. Generally, the results were best in the patients with the least advanced arthritic changes. Even in grade IV knees, $44.8 \%$ ( $41.2 \%$ by KSS for pain) of the patients were satisfied with this procedure. Although this outcome is not as good as that provided by total knee replacement, it still offers a benefit for many patients because of its simplicity and low morbidity.

The outcomes of commonly-used arthroscopic procedures for osteoarthritis of the knee, including joint lavage, debridement, abrasion arthroplasty and microfracture, are inconsistent. In most patients, short-term symptomatic relief can be expected with arthroscopic lavage and debridement. ${ }^{11}$ In 2002, Moseley et $\mathrm{al}^{8}$ performed a double-blind, randomised, placebo-controlled trial to compare the effectiveness of arthroscopic lavage and arthroscopic debridement versus a sham procedure and found that arthroscopy provided subjective pain relief via a placebo effect. Abrasion chondroplasty ${ }^{5}$ was popularised in the late 1980s. Rand, ${ }^{10}$ comparing arthroscopic partial meniscectomy with limited debridement and arthroscopic abrasion arthroplasty in patients with osteoarthritis, found that abrasion arthroplasty offered little benefit over partial meniscectomy and debridement. Concern exists over the durability of the fibrocartilage repair tissue in subchondral penetration procedures, and over thermal damage to subchondral bone and adjacent normal articular cartilage during this procedure. ${ }^{1}$ Microfracture is a modification of chondroplasty, but a deterioration of the results has been reported starting 18 months after surgery. ${ }^{6}$

The concept of medial release procedures is similar. The main purpose of Loeffler's operation ${ }^{20}$ is to release the tight medial soft-tissue structures and excise hypertrophied synovial tissue, which may cause pain in degenerative knees. Leon et $\mathrm{al}^{24}$ and Moriya et $\mathrm{al}^{25}$ claim that their procedures of arthroscopic release unload the medial compartment by releasing the medial capsule and medial collateral ligament, which may permit a reduced adduction moment and a reduction of the external rotation restraint in extension found in more severely osteoarthritic knees.
The main rationale for our arthroscopic medial release is to eradicate the abrasion or impingement phenomenon between the tight, fibrotic and hypertrophied medial plica and the adjacent medial femoral condyle, which has been described in previous studies in patients with osteoarthritic knees. ${ }^{26,31,32}$ It has been reported that certain neuropeptides are responsible for the neural transmission of pain in the synovium and capsule of osteoarthritic knees. ${ }^{33}$ This may account for most of our patients obtaining early, rapid pain relief. This effect has also been reported by Ikeuchi, Takanashi and Tani, ${ }^{34}$ who successfully treated 19 osteoarthritic knees with localised synovial hypertrophy in the anteromedial compartment by arthroscopic partial synovectomy. In a one-year longitudinal arthroscopic study in 422 patients, Ayral et $\mathrm{al}^{35}$ found that abnormalities of the medial synovium were a common feature of painful medial osteoarthritis of the knee associated with more marked medial chondropathy. In addition, they suggested that an inflammatory aspect of the medial perimeniscal synovium could be related to a subsequent medial degradation. Therefore, the eradication of all the inflammatory synovium in this area in our procedure might have reduced or arrested the degenerative process in the medial compartment.

The prevalence of a medial plica varies from $19 \%$ to $95 \%{ }^{36,37}$ The universal presence of the medial plica in our patients with osteoarthritic knees, and the relationship between its size and gross appearance and the patients' age, are in keeping with a previous study. ${ }^{26}$ Whether the medial plica plays a role in the pathogenesis of the osteoarthritis is worthy of further investigation.

A limitation of this study was the lack of radiological follow-up evaluation to correlate with the clinical outcome. The lack of independence in the grading system and no measures of reproducibility were also drawbacks of this study.

In conclusion, this experience with arthroscopic medial release suggests that it can reduce pain in the majority of patients with medial compartment osteoarthritis of the knee over a period of at least four years. Whether it is able to modify the disease process warrants more prospective studies, including imaging evaluation. The high incidence of a medial plica in patients with medial compartment degenerative disease also justifies further investigations for its role of this structure in the pathogenesis of osteoarthritis.

\section{Supplementary Material}

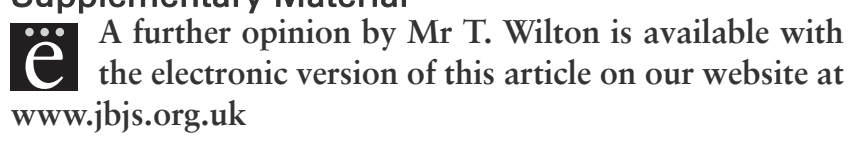

No benefits in any form have been received or will be received from a commercial party related directly or indirectly to the subject of this article.

\section{References \\ 1. Hunt SA, Jazrawi LM, Sherman $\mathbf{O H}$. Arthroscopic management of osteoarthritis of the knee. J Am Acad Orthop Surg 2002;10:356-63.}


2. Wai EK, Kreder HJ, Williams Jl. Arthroscopic debridement of the knee for osteoarthritis in patients fifty years of age or older: utilization and outcomes in the Province of Ontario. J Bone Joint Surg [Am] 2002;84-A:17-22.

3. Day B. The indications for arthroscopic debridement for osteoarthritis of the knee. Orthop Clin North Am 2005;36:413-17.

4. Kelly MA. Role of arthroscopic debridement in the arthritic knee. J Arthroplasty 2006;21(4 Suppl 1):9-10.

5. Dandy DJ. Abrasion chondroplasty. Arthroscopy 1986;2:51-3.

6. Bae DK, Yoon KH, Song SJ. Cartilage healing after microfracture in osteoarthritic knees. Arthroscopy 2006;22:367-74.

7. Kreuz PC, Steinwachs MR, Erggelet C, et al. Results after microfracture of fullthickness chondral defects in different compartments in the knee. Osteoarthritic Cartilage 2006;14:1119-25.

8. Moseley JB, O'Malley K, Petersen NJ, et al. A controlled trial of arthroscopic surgery for osteoarthritis of the knee. N Engl J Med 2002;347:81-8.

9. Bernstein J, Quach T. A perspective on the study of Moseley et al: questioning the value of arthroscopic knee surgery for osteoarthritis. Cleve Clin J Med 2003;70:401,4056,408-10.

10. Rand JA. Role of arthroscopy in osteoarthritis of the knee. Arthroscopy 1991;7:358-63.

11. Shannon FJ, Devitt AT, Poynton AR, Fitzpatrick P, Walsh MG. Short-term benefit of arthroscopic washout in degenerative arthritis of the knee. Int Orthop 2001;25:242-5.

12. Siparsky P, Ryzewicz M, Peterson B, Bartz R. Arthroscopic treatment of osteoarthritis of the knee: are there any evidence-based indications? Clin Orthop 2007;455:10712.

13. Haggart GE. Surgical treatment of degenerative arthritis of the knee joint. J Bone Joint Surg 1940;22:717-29

14. Magnuson PB. Technic of debridement of the knee joint for arthritis. Surg Clin North Am 1946;26:249-66.

15. Insall J. Intra-articular surgery for degenerative arthritis of the knee: a report of the work of the late K.H. Pridie. J Bone Joint Surg [Br] 1967;49-B:211-28.

16. Insall J. The Pridie debridement operation for osteoarthritis of the knee. Clin Orthop 1974;101:61-7.

17. MacIntosh DL, Welsh RP. Joint debridement: a complement to high tibial osteotomy in the treatment of degenerative arthritis of the knee. J Bone Joint Surg [Am] 1977;59A:1094-7.

18. Ha'eri GB, Wiley AM. High tibial osteotomy combined with joint debridement: a longterm study of results. Clin Orthop 1980;151:153-9.

19. McEldowney AJ, Weiker GG. Open-knee Magnuson debridement as conservative treatment for osteoarthritis of the knee. J Arthroplasty 1995;10:805-9.
20. Loeffler F. The operative treatment of severe painful orthoses of the knee joint. Zentralb/ Chir 1960;85:1020-3 (in German).

21. Reiter R. Loeffler's operation of the knee joint. Z Orthop Ihre Grenzgeb 1965;100:345-50 (in German).

22. Reichel F, Muller-Stephann H. Loeffler's capsulotomy in advanced varus gonarthrosis. Beitr Orthop Traumatol 1988;35:453-7 (in German).

23. Schonbauer HR. Experience and results with the Loeffler operation for osteoarthritis of the knee joint. Arch Orthop Unfallchir 1976;85:337-46 (in German).

24. Leon HO, Blanco CER, Guthrie TB. Arthroscopic decompressive medial release of the varus arthritic knee: expanding the functional envelope. Arthroscopy2001;17:5236.

25. Moriya H, Sasho T, Sano S, Wada Y. Arthroscopic posteromedial release for osteoarthritic knees with flexion contracture. Arthroscopy 2004;20:1030-9.

26. Lyu SR, Hsu CC. Medial plicae and degeneration of the medial femoral condyle. Arthroscopy 2006;22:17-26.

27. Marx RG, Connor J, Lyman S, et al. Multirater agreement of arthroscopic grading of knee articular cartilage. Am J Sports Med 2005;33:1654-7.

28. Kellgren JH, Lawrence JS. Atlas of standard radiographs: the epidemiology of chronic rheumatism. Vol. 2. Oxford: Blackwell, 1963

29. Insall JN, Dorr LD, Scott RD, Scott WN. Rationale of the Knee Society clinical rating system. Clin Orthop 1989;248:13-14.

30. Warren LF, Marshall JL. The supporting structures and layers on the medial side of the knee: an anatomical analysis. J Bone Joint Surg [Am] 1979;61-A:56-62.

31. Lyu SR, Tzeng JE, Kuo CY, Jian AR, Liu DS. Mechanical strength of mediopatellar plica: the influence of its fiber content. Clin Biomech 2006;21:860-3.

32. Lyu SR. Relationship of medial plica and medial femoral condyle during flexion. Clin Biomech 2007;22:1013-16.

33. Saito T, Koshini T. Distribution of neuropeptides in synovium of the knee with osteoarthritis. Clin Orthop 2000;376:172-82.

34. Ikeuchi M, Takanashi T, Tani T. Localized synovial hypertrophy in the anteromedial compartment of the osteoarthritic knee. Clin Orthop 2005;21:1457-61.

35. Ayral X, Pickering EH, Woodworth TG, Mackillop N, Dougados M. Synovitis: a potential predictive factor of structural progression of medial tibiofemoral knee osteoarthritis: results of a 1 year longitudinal arthroscopic study in 422 patients. Osteoarthritis Cartilage 2005;5:361-7.

36. Dupont JY. Synovial plicae of the knee: controversies and review. Clin Sports Med 1997;16:87-122.

37. Gurbuz H, Calpur OU, Ozcan M, Kutoglu T, Mesut R. The synovial plicae in the knee joint. Saudi Medical Journal 2006;27:1839-42. 